\title{
SYMBIOTIC BINARY STARS
}

\author{
SCOTT J. KENYON \\ Harvard-Smithsonian Center for Astrophysics 60 Garden Street, Cambridge, MA 02138
}

\begin{abstract}
This paper briefly reviews the physical properties of symbiotic stars: long-period interacting binaries composed of a red giant primary star and a hot companion. Two types of binaries produce symbiotic optical spectra: semi-detached systems with a main sequence secondary and detached systems with a white dwarf secondary. Semi-detached symbiotics resemble cataclysmic variables and Algol binaries, but on a much larger scale, and undergo dwarf nova-like eruptions. Wind accretion powers detached systems; occasional thermonuclear runaways produce symbiotic novae - distant cousins of classical novae.
\end{abstract}

Key words: stars: binaries: symbiotics - accretion discs - stars: mass loss

\section{Introduction}

Throughout the compilation of the HD catalog, A. Cannon and W.P. Fleming discovered several bright red giant stars with unusually strong hydrogen and helium emission lines (see Fleming 1912). Most of these stars with combination spectra showed 0.5-1 mag brightness variations on time scales of months to years, while several had undergone 2-4 mag eruptions lasting several years. P. Merrill, M.Humason, and several others had identified roughly two dozen stars with combination spectra by 1944 , when Merrill suggested symbiotic stars to describe objects displaying cool stellar absorption features and strong nebular emission lines.

The class of symbiotic variable stars now includes over 150 objects in our own galaxy and a handful of systems in other Local Group galaxies. Most symbiotics lie close to the galactic plane, and their scale height and velocity dispersion suggest membership in the intermediate or old disc population (Wallerstein 1981; Duerbeck 1984). Although most galactic symbiotics contain red giant stars or Mira variables, most extragalactic ones contain carbon stars (see Allen 1984, 1988; Kenyon 1986). The large population of extragalactic symbiotic carbon stars - especially in the Magellanic Clouds - parallels the relative numbers of field carbon stars in our galaxy and in the Clouds (Blanco et al. 1978).

My definition of symbiotic stars relies on type examples that includes Merrill's classical symbiotic stars - Z And, BF Cyg, CI Cyg, RW Hya, AG Peg, and AX Per - and some recent symbiotic novae - V1016 Cyg, V1329 Cyg, HM Sge, and PU Vul - that will resemble the classical systems once they return to quiescence (see also Nussbaumer's discussion of symbiotic novae in this volume). Quiescent and a few eruptive systems generally show red giant absorption features - $\mathrm{Ca} \mathrm{I}, \mathrm{H}_{2} \mathrm{O}, \mathrm{CO}$, and $\mathrm{TiO}$, a bright blue continuum, and high ionization emission lines - H I, He I, He II, [O III], and sometimes [Ne V] and [Fe VII] (Fig. 1). Many eruptive systems more closely resemble classical novae and display an $\mathrm{A}$ or $\mathrm{F}$ supergiant absorption spectrum at visual maximum (Kenyon 1986). Optical and infrared photometric variations of $\lesssim 1 \mathrm{mag}$ also seem characteristic of many symbiotics, but the historical records for most systems are very incomplete. 


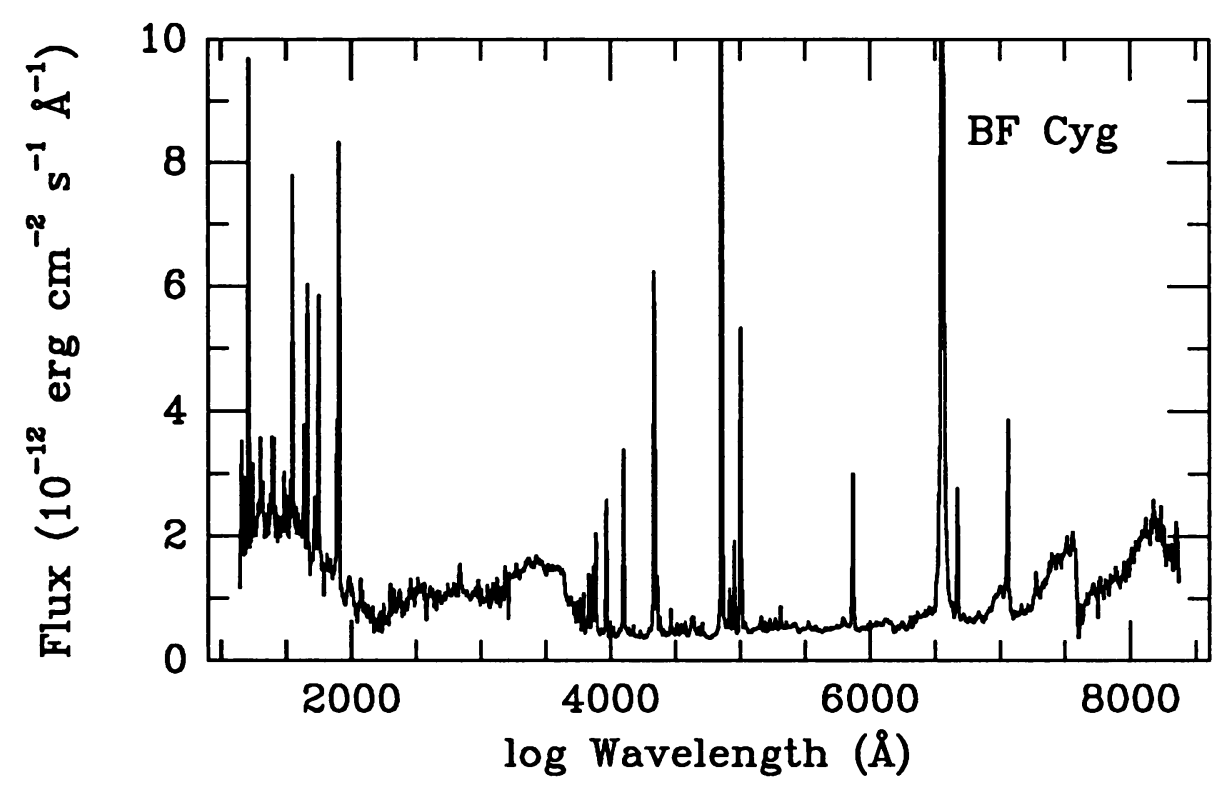

Figure 1. Optical/ultraviolet spectrum for BF Cygni. This spectrum shows the Rayleigh-Jeans continuum from the hot component, prominent emission lines and Balmer Jump from the nebula, and $\mathrm{TiO}$ absorption bands from the red giant.

\section{Are symbiotics binary stars?}

An interacting binary system naturally explains the spectroscopic peculiarities of symbiotic stars, because the two observed temperature extremes can be associated with separate stellar objects. Berman (1932) and Hogg (1934) suggested that an M giant with a faint O- or B-type companion star might account for the optical spectrum. They attributed small photometric fluctuations to binary motion and associated larger eruptions with unidentified instabilities in the hot star itself. Kuiper (1941) later recommended an alternative binary model in which a Roche-lobe filling red giant transferred material onto a low mass main sequence star. In this hypothesis, outbursts and smaller amplitude fluctuations are caused by modulations of the mass transfer rate, rather than physical changes in the structure of the hot star.

Various astronomers developed single star models when observers failed to identify orbital motion in some symbiotic stars (e.g., Menzel 1946; Aller 1954; Wood 1973; Hack and Selvelli 1982). However, recent ground-based and satellite observations support the binary interpretation. For example, eclipses of the hot component by the giant simply account for periodic optical photometric variations in CI Cyg and AX Per (Kenyon et al. 1991; Mikolajewska and Kenyon 1992a). Other periodic photometric variations - as observed in AG Peg - seem caused by the reflection effect, where the hot component heats up the facing hemisphere of the giant (see Kenyon 1986; Sec. 3.2).

Radial velocity measurements also convincingly demonstrate that symbiotics are binary stars. Merrill, Struve, Swings, and other observers had problems proving binary motion for many symbiotics in the 1940's and 1950's, because the orbital 


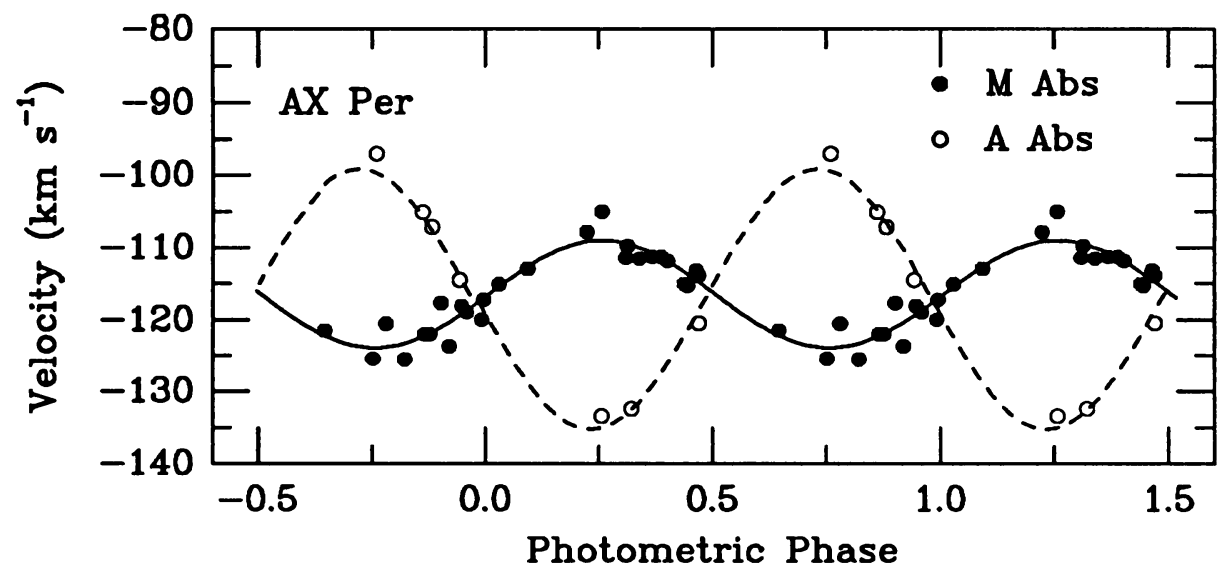

Figure 2. Radial velocity curves for AX Per. The solid points plot $M$ giant velocities, while open circles plot velocities of the A-type hot component in outburst. The best fitting orbital solutions shown as solid and dashed lines imply a mass ratio of $q=M_{g} / M_{h} \sim 2.5$.

amplitudes were small - 5-10 $\mathrm{km} \mathrm{s}^{-1}$ - and 1-3 mag optical eruptions were frequent. However, modern observations with echelle spectrographs and solid state detectors provide high quality velocity measurements capable of verifying orbital motion in 1-2 orbital cycles (Fig. 2). Table 1 summarizes reliable orbital solutions for a small sample of symbiotics observed with the 1.5-m telescopes at Mt. Hopkins, Arizona and Harvard, Massachusetts (see, for example, Kenyon and Garcia 1989). In most cases, these orbits suggest the red giant is the more massive binary component, with $M_{g} \sim 1-3 \mathrm{M}_{\odot}$ and $M_{h} \sim 0.5-1.5 \mathrm{M}_{\odot}$.

The International Ultraviolet Explorer delivered a fatal blow to single star hypotheses in the 1980's, when it detected a strong blue continuum in nearly every bright symbiotic star. The spectrum of BF Cyg in Fig. 1 displays prominent Rayleigh-Jeans and recombination continua - including a Balmer emission jump and intense emission lines in addition to the red continuum and absorption lines from the giant. The rising blue continuum and high ionization emission lines suggest the hot component possesses an optically thick photosphere with a temperature exceeding $5 \times 10^{4} \mathrm{~K}$.

Fig. 1 shows that the three components of a typical symbiotic system dominate the energy distribution at specific wavelengths. This fact is both a blessing and a curse: a well-defined observational program can probe a particular component of a symbiotic binary, but progress in understanding the binary system requires a complete spectral energy distribution. To this end, I describe the basic properties of the hot components, cool components, and nebular regions in the next three sections, and then combine these features into a simple binary picture in Sec. 6 .

\section{The hot component}

The hot component captures the attention of most symbiotic scientists because it powers the observed activity, such as small night-to-night fluctuations in emission 
Table 1. Orbital solutions for symbiotic stars

\begin{tabular}{lccccc}
\hline \hline Symbiotic & $\mathrm{P}_{\text {orb }}($ days $)$ & $\gamma\left(\mathrm{km} \mathrm{s}^{-1}\right)$ & $\mathrm{K}\left(\mathrm{km} \mathrm{s}^{-1}\right)$ & $e$ & $f\left(M_{g}, M_{h}\right)$ \\
\hline EG And & 480.7 & -95.0 & 7.5 & $0.043 \pm 0.021$ & 0.021 \\
Z And & 756.9 & 2.6 & 6.9 & $0.20 \pm 0.11$ & 0.024 \\
UV Aur & 395.2 & & 7.4 & $0.29 \pm 0.20$ & 0.015 \\
TX CVn & 199.0 & 2.3 & 5.7 & $0.16 \pm 0.06$ & 0.004 \\
T CrB & 227.5 & -27.9 & 23.3 & $0.012 \pm 0.005$ & 0.299 \\
CI Cyg & 855.3 & 18.3 & 7.0 & $0.15 \pm 0.08$ & 0.029 \\
AG Dra & 554.0 & -146.1 & 5.2 & $0.18 \pm 0.32$ & 0.008 \\
V443 Her & 600.0 & -49.4 & 3.3 & $0.18 \pm 0.26$ & 0.002 \\
RW Hya & 372.5 & 14.6 & 8.0 & 0 & 0.020 \\
AG Peg & 818.7 & -14.4 & 6.1 & $0.12 \pm 0.04$ & 0.019 \\
AX Per & 680.8 & -116.2 & 7.6 & $0.12 \pm 0.15$ & 0.030 \\
AS 296 & 600.0 & & 6.6 & 0 & 0.017 \\
\hline \hline
\end{tabular}

line fluxes and the more prominent 2-7 mag eruptions. Aside from the Balmer emission jump and various emission lines, the quiescent hot components emit most of their radiation below $3200 \AA$, where only satellites observe.

Two groups have systematically analyzed the UV continua and emission line fluxes from a representative sample of $20-25$ objects (Kenyon and Webbink 1984; Mürset et al. 1991). Although the details in their analyses differ somewhat, both studies conclude that the hot components in most symbiotic binaries are compact stars resembling the central stars of planetary nebulae. The effective temperatures of these "hot subdwarfs" generally range from $T_{h} \sim 3-5 \times 10^{4} \mathrm{~K}$ to $T_{h} \sim 2 \times 10^{5}$ K. Most quiescent symbiotics have luminosities of $L_{h} 10-1000 \mathrm{~L}_{\odot}$, while eruptive systems reach $L_{h} \sim 3 \times 10^{4} \mathrm{~L}_{\odot}$. Thus, their radii are $R_{h} \sim 0.01-10 \mathrm{R}_{\odot}$.

Many eruptive systems have been called "symbiotic novae" and "very slow novae", because they behave like classical novae but on a much longer time scale (Allen 1980; Kenyon and Truran 1983). (Payne-Gaposchkin (1957) invented "symbiotic novae" to describe the entire class, but it is now reserved for those symbiotic stars that undergo slow nova-like eruptions). These systems increase in optical brightness by 3-7 mag over several years, and they maintain a constant bolometric luminosity during their slow declines from visual maximum (see Nussbaumer, this volume). The main difference between classical novae and symbiotic novae is the nature of the eruptive white dwarf's companion: classical novae contain lobe-filling red dwarfs while symbiotic novae contain detached red giants (see Kenyon 1986).

Several other hot white dwarfs appear to maintain a constant luminosity at lower levels than the symbiotic novae. Mikolajewska, Kenyon, and Mikolajewski (1989) suggested that BF Cyg - and perhaps also Z And, V443 Her, RW Hya, and SY Mus (see Mikolajewska and Kenyon 1992b) - contains a 0.5-0.6 $\mathrm{M}_{\odot}$ white dwarf steadily burning material accreted from a red giant wind. Eruptions in these systems presumably occur when the accretion rate changes, but the predicted rise and decay times exceed those observed by several orders of magnitude. 
Kenyon and Webbink (1984) suggested that the UV continua observed in several systems more closely resemble the energy distributions of accretion discs surrounding solar-type main sequence stars than of single temperature stars. Mürset et al. (1991) dispute this proposal and conclude that hot white dwarfs can also explain the energy distributions of CI Cyg, YY Her, and AX Per. However, time variations of the UV and optical fluxes in CI Cyg and AX Per identify the hot component as an accreting main sequence star, rather than a hot white dwarf. For example, the equatorial radius of the hot component in $\mathrm{AX}$ Per $-R_{e} \sim 25 \mathrm{R}_{\odot}$ - exceeds the spherically symmetric radius of $R_{s} \lesssim 12 \mathrm{R}_{\odot}$ estimated from the bolometric luminosity (Mikolajewska and Kenyon 1992a). If we approximate the hot component as an ellipsoid, then its major axis is at least 4 times larger than its minor axis. This result - along with a wavelength dependent spectral type and the rough equivalence of the extreme UV and UV/optical luminosities - point to accretion-powered eruptions with a main sequence central star surrounded by a hot, thin boundary layer and a large circumstellar disc (see Kenyon et al. 1991 and Mikolajewska and Kenyon 1992a for more detailed descriptions of CI Cyg and AX Per).

Aside from accreting main sequence stars and hot white dwarfs, at least one symbiotic binary - V2116 Oph $=\mathrm{GX1}+4$ - contains an accreting neutron star. Unfortunately, the large extinction to V2116 Oph $-A_{V} \sim 8 \mathrm{mag}$ - precludes detailed optical or UV studies. A related X-ray binary - HD 154791 - does not produce strong optical emission lines, but Garcia et al. (1983) found strong C IV and N V emission lines on IUE spectra. The lower X-ray luminosity in HD 154791 $L_{X} \sim 10^{33}$ to $10^{34} \mathrm{erg} \mathrm{s}^{-1}$ versus $5 \times 10^{38} \mathrm{erg} \mathrm{s}^{-1}$ in V2116 Oph - probably cannot photoionize a large enough nebula to produce optical emission lines.

The preceding discussion requires that some symbiotic stars are interacting binaries, because mass transfer from the giant energizes the hot component when the secondary is a main sequence star or a neutron star. However, mass transfer must also power those symbiotics with white dwarf secondaries. For example, the $\sim 10^{5}$ yr cooling time for a $0.6 \mathrm{M}_{\odot}$ white dwarf with $L_{h} \sim 100 \mathrm{~L}_{\odot}$ (e.g., Paczyński 1976 ) is much shorter than the $\sim 10^{7} \mathrm{yr}$ lifetime of a typical low mass red giant star. Thus, a newly-formed hot white dwarf (the ex-primary) cools to $T_{h} \sim 10^{4} \mathrm{~K}$ before its companion evolves to red giant dimensions unless the initial mass ratio is very close to unity. Most binaries are not equal mass pairs, so the white dwarfs in symbiotic binaries must be rejuvenated by material accreted from the red giant stellar wind to reach high effective temperatures.

\section{The cool component}

Infrared observations have provided most of our information about the cool components in symbiotic stars, because red giants emit most of their radiation between $0.7 \mu \mathrm{m}$ and $5 \mu \mathrm{m}$. Infrared colors, such as $\mathrm{H}-\mathrm{K}$ and $\mathrm{K}-\mathrm{L}$, neatly divide symbiotics into $S$-type (stellar) systems with the color temperatures of normal red giants, 2500-3500 K, and D-type (dusty) systems with much lower color temperatures, $1000 \mathrm{~K}$ (Webster and Allen 1975). The cool components in S-type systems tend to be non-variable $(\Delta K \lesssim 0.1-0.2 \mathrm{mag}$ ) red giant stars with moderately strong $C O$ absorption bands at $2.3 \mu \mathrm{m}$ and modest IR excesses at wavelengths exceeding 10 
$\mu \mathrm{m}$. The D-type systems, on the other hand, contain very evolved Mira variables ( $\Delta K \sim 1$ mag; $\mathrm{P} \sim 300-700$ days) with strong $\mathrm{CO}$ and $\mathrm{H}_{2} \mathrm{O}$ absorption bands on their $2 \mu \mathrm{m}$ spectra and large 10-20 $\mu \mathrm{m}$ excesses.

Most observations suggest the red giant components lose mass at the high rates required to power the hot components. The D-type symbiotics easily achieve high mass loss rates, because single Miras commonly lose mass at rates of $\dot{\mathrm{M}}_{\mathrm{g}} \gtrsim$ $10^{-7} \mathrm{M}_{\odot} \mathrm{yr}^{-1}$ (Dupree 1986). Whitelock (1987) and Kenyon, Fernández-Castro, and Stencel (1988) estimated $10^{-7} \mathrm{M}_{\odot} \mathrm{yr}^{-1} \lesssim \dot{\mathrm{M}}_{\mathrm{g}} \lesssim 10^{-5} \mathrm{M}_{\odot} \mathrm{yr}^{-1}$ from 12-100 $\mu \mathrm{m}$ data acquired by the Infrared Astronomical Satellite, and Seaquist and Taylor (1990) confirmed these results with radio continuum measurements at $2-20 \mathrm{~cm}$.

For a neutral wind with $\dot{\mathrm{M}}_{\mathrm{g}} \sim 10^{-5} \mathrm{M}_{\odot} \mathrm{yr}^{-1}$ and a velocity of $100 \mathrm{~km} \mathrm{~s}^{-1}$, the local optical reddening should be $A_{V} \sim 10-100 \mathrm{mag}$ (Mikolajewska and Kenyon 1992b). Whitelock (1987) and Kenyon, Fernández-Castro, and Stencel (1988) estimated infrared extinctions of $A_{K} \sim 1-2$ mag for many symbiotics, which implies $A_{V} \sim 10-20 \mathrm{mag}$ for a normal extinction law (Savage and Mathis 1979). Thus, local extinction in the dense Mira wind produces the very cool infrared color temperatures of $\sim 1000 \mathrm{~K}$ observed in the D-type symbiotics.

Some S-type systems lose mass rapidly via Roche Lobe overflow. Optical eclipse timings suggest both main sequence accretors - CI Cyg and AX Per - fill their tidal surfaces for reasonable ranges in the orbital inclination (Kenyon et al. 1991; Mikolajewska and Kenyon 1992a). AR Pav probably also loses mass tidally, but its light curve outside eclipse has irregular variations that remain unexplained.

Other S-type systems reach high $\dot{M}$ 's without tidal overflow. Seaquist and Taylor (1990) and Kenyon, Fernández-Castro, and Stencel (1988) find that systems with white dwarf secondaries lie near the upper envelope of measured $\dot{M}$ 's for normal red giants even though many of them have radii, $R_{g}$, much smaller than their Roche lobe radii, $R_{L}$. Tout and Eggleton (1988) suggested mass loss from the red giant in a symbiotic binary might still be enhanced by tidal interaction even if $R_{g} / R_{L} \sim 1 / 3$ to $1 / 2$. Mikołajewska, Kenyon, and Mikolajewski (1989) tried to verify enhanced mass loss in BF Cyg, and the observed system $\dot{M}$ seems to confirm Tout and Eggleton's predictions. However, more tests are needed.

Finally, eclipse studies offer the possibility of deriving mass loss rates and velocity laws for red giant winds. Isliker, Nussbaumer, and Vogel (1989) showed that Rayleigh scattering by material in the wind removes short wavelength UV photons from our line-of-sight and produce UV energy distributions that agree with observations. Vogel (1991) applied this model to EG And and estimated a mass loss rate of $1-2 \times 10^{-8} \mathrm{M}_{\odot} \mathrm{yr}^{-1}$ for an adopted terminal velocity of $30 \mathrm{~km} \mathrm{~s}^{-1}$. He finds the wind accelerates to terminal velocity at somewhat larger distances - 3-9 red giant radii - than assumed by "standard" velocity laws.

\section{The nebula}

Most symbiotic stars display bright emission lines similar to those observed in planetary nebulae, and these features reflect the intense radiation field emitted by the hot component. Strong H I and He I lines usually appear on optical spectra, while $\mathrm{Mg}$ II, C III], O III], and C IV lines are visible on most ultraviolet spectra 
(see Fig. 1). The more extreme symbiotics present additional lines from $\mathrm{He}$ II, NV, $[\mathrm{Ne} \mathrm{V}],[\mathrm{Fe} \mathrm{VII}]$, and the enigmatic $\lambda 6830$ band.

Many ultraviolet emission line fluxes can probe physical conditions within symbiotic nebulae, and we can develop a pretty good snapshot of a given system when simultaneous optical spectrophotometry supplements IUE spectra (see Nussbaumer and Stencel 1987). These diagnostics divide symbiotics into objects with high electron densities, $n_{e} \sim 10^{8}$ to $10^{10} \mathrm{~cm}^{-3}$, and lower density systems with $n_{e} \sim 10^{5}$ to $10^{7} \mathrm{~cm}^{-3}$ (Nussbaumer and Stencel 1987). Dense nebulae generally inhabit S-type symbiotics, while D-type systems typically possess lower density nebulae. The electron temperatures in S- and D-types, $T_{e} \sim 1-4 \times 10^{4} \mathrm{~K}$, suggest the gas is photoionized and not shock-excited. However, the main sequence accretors CI Cyg and AX Per produce [Fe VII] emission from a mechanically heated region having a low density, $n_{e} \sim 10^{7} \mathrm{~cm}^{-3}$, and a high electron temperature, $T_{e} \gtrsim 10^{5}$ K (Kenyon et al. 1991; Mikolajewska and Kenyon 1992a). Willson et al. (1984) proposed that shock-excited X-ray and line emission could be produced in symbiotic novae when the wind from the hot component collides with the red giant wind.

Nussbaumer et al. (1988) presented estimates for the elemental abundances, $\mathrm{O} / \mathrm{N}$ and $\mathrm{C} / \mathrm{N}$, in symbiotic nebulae based on $I U E$ spectra and detailed nebular models. They find $\mathrm{O} / \mathrm{N}$ and $\mathrm{C} / \mathrm{N}$ to be much smaller than the solar abundance but similar to abundances observed in normal red giants. Thus, the ionized gas appears to be nitrogen-rich material produced by evolutionary processes within the red giant and then ejected. This hypothesis could be tested by comparing abundances in the cool components with nebular abundances.

Line profiles contain useful information concerning mass motions in symbiotic binaries. Several groups have monitored $\mathrm{H} \alpha$ and other strong emission lines in symbiotic novae and a few of the brighter classical symbiotics, but these data are hard to interpet (see Kenyon 1986). Most lines appear to contain several components that behave differently with time; some of these variations correlate with the orbital period but others do not.

\section{What binaries become symbiotic?}

The data gathered from ground-based and satellite observatories during the past two decades confirm the simple binary models proposed by Berman, Hogg, and Kuiper in the 1930's and 1940's. These data suggest that symbiotic stars can be divided into three broad classes of objects, as illustrated in Fig. 3.

Some symbiotic stars are closely related to other types of interacting binaries and contain a lobe-filling red giant primary and a main sequence star secondary. The prototypical examples of semi-detached symbiotic binaries are CI Cyg $\left(P_{\text {orb }}=855\right.$ days) and AX Per $\left(P_{\text {orb }}=681\right.$ days $)$, and Fig. 3 shows a schematic representation of their binary components. In both systems, the red giant fills or nearly fills its tidal surface and eclipses a hot component that is extended in the orbital plane. A disc provides the most natural interpretation for the geometrical shape of the hot component and its energy distribution. The temperature and luminosity of the disc and a hot boundary layer at the disc's inner edge suggest the accreting object is a main sequence star in both systems. 

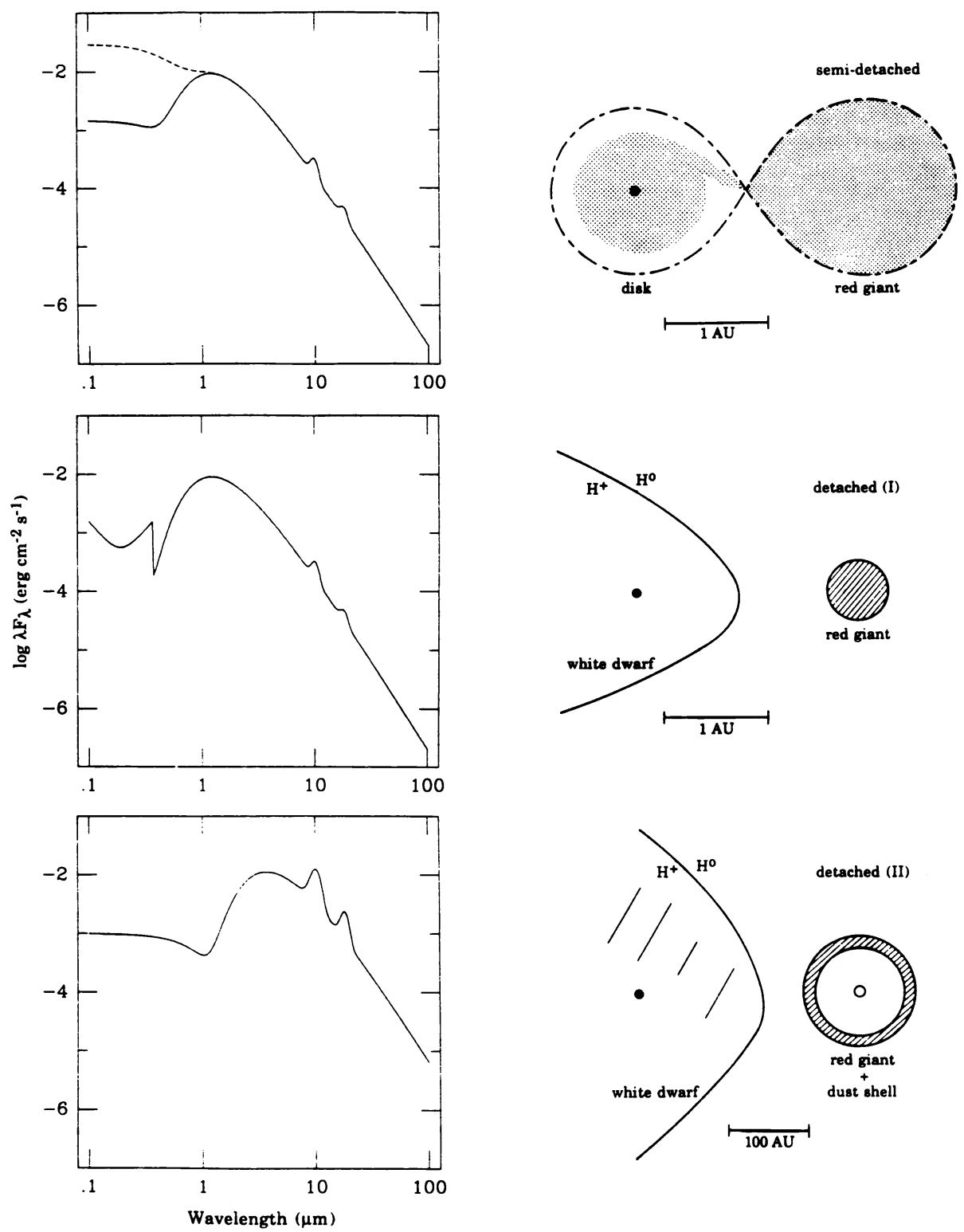

Figure 3. Schematic illustrations of the energy distribution (left panels) and system geometry (right panels) for the three types of symbiotic binaries described in the text. 
The upper left panel of Fig. 3 shows an illustrative spectral energy distribution for a system like CI Cyg. During quiescent periods, the red giant star produces most of the energy received at wavelengths exceeding $0.5 \mu \mathrm{m}$ (solid line); the accretion disc is responsible for radiation at shorter wavelengths and the intense emission lines (the emission lines have been left off the energy distributions for clarity). Most emission lines are produced in a photoionized region confined to the Roche volume of the hot star; some high ionization lines form in a shock-heated bipolar structure at larger distances from the disc (see Kenyon et al. 1991).

The accretion disc brightens dramatically during an eruption. The mass flow rate through the disc rises abruptly from $\sim 10^{-5} \mathrm{M}_{\odot} \mathrm{yr}^{-1}$ to $\sim 10^{-3} \mathrm{M}_{\odot} \mathrm{yr}^{-1}$ and declines in 1-3 years. Aside from an obvious change in the energy distribution (indicated by the dashed line in the Figure), the optical spectrum evolves into a configuration resembling an $\mathrm{A}$ or $\mathrm{F}$ supergiant star with no evidence for high ionization emission lines. The physical cause of the outburst is not known with certainty, but the disc brightens probably because the mass loss rate of the giant increases or the disc is thermally unstable (see Duschl 1988 and references therein).

The most abundant type of symbiotic binary contains a red giant star that underfills its inner Lagrangian surface and loses material via a stellar wind. Good examples of this class are RW Hya (a quiescent system; $P_{\text {or } b} \sim 372$ days) and AG Peg (an erupting system; $P_{\text {orb }} \sim 818$ days). The middle right panel of Fig. 3 shows the binary geometry, while the middle left panel plots a typical energy distribution. As in the semi-detached systems, the red giant produces most of the long wavelength emission, while the Rayleigh-Jeans tail of the hot white dwarf and Balmer continuum radiation from the ionized nebula dominate at shorter wavelengths.

Roughly $20 \%$ of all known symbiotic binaries contain Mira variables instead of non-pulsating red giant stars. Good examples of this type of system are V1016 Cyg and H1-36. These objects possess the strongest high ionization emission lines and the largest ionized nebulae among symbiotic stars, because their hot white dwarfs generally outshine those in their shorter period siblings. I suspect that Dtype symbiotics are those Mira + white dwarf binaries in which the white dwarf has undergone a symbiotic nova eruption; these systems might resemble Mira Ceti when the white dwarf is in a quiescent stage (see Mikolajewska et al. 1988).

Although we do not know the orbital period of any Mira symbiotic, the radio observations suggest large binary separations ( 10-100 AU; Seaquist and Taylor 1990). Optically thick dust shells also distinguish D-type systems from S-type systems, as illustrated in the lower right panel of Fig. 3. The local extinction in the D-types reddens their near-IR energy distributions (Fig. 3; lower left panel). This extra extinction proably is caused by an optically thick dust shell which surrounds the Mira and has a radius of $\sim 30 \mathrm{AU}$ (assuming spherical symmetry).

If the core mass-luminosity relation holds for the white dwarfs in symbiotic binaries, then their masses exceed those found among field white dwarfs. Mikolajewska and Kenyon (1992b; see also Nussbaumer, this volume) estimated masses of 0.5$0.6 \mathrm{M}_{\odot}$ for a handful of systems, but many symbiotic novae contain more massive white dwarfs with $\mathrm{M} \sim 0.8-1.1 \mathrm{M}_{\odot}$. The slow eruptions of these systems suggest that the white dwarfs gain mass with time and might eventually explode as type I supernovae. Iben and Tutukov (1984) estimated that symbiotic stars might produce 
symbiotic novae at a rate of 5 per $1000 \mathrm{yr}$. This rate is close to the observed type I rate of 5-15 per 1000 yr (van den Bergh and Tammann 1991).

\section{References}

Allen, D.A. 1980. Mon. Not. Roy. Astr. Soc., 190, 75.

Allen, D.A. 1983. Mon. Not. Roy. Astr. Soc., 204, 113.

Allen, D.A. 1984. Publ. Astr. Soc. Aus., 35, 369.

Allen, D.A. 1988. In The Symbiotic Phenomenon, IAU Colloquium No. 103, edited by J. Mikołajewska, et al. (Kluwer, Dordrecht), p. 3.

Aller, L.H. 1954. In Astrophysics - Nuclear Transformations, Stellar Interiors, and Nebulae (New York: Ronald), p. 180.

Berman, L. 1932. Publ. Astr. Soc. Pac., 44, 318.

Blanco, B.M., Blanco, V.M., and McCarthy, M.F. 1978. Nature, 271, 638.

Duerbeck, H.W. 1984. Astrophys. Space Sci., 99, 363.

Dupree, A.K. 1986. Ann. Rev. Astr. Ap., 24, 377.

Duschl, W.J. 1988. In The Symbiotic Phenomenon, IAU Colloquium No. 103, edited by J. Mikołajewska, et al. (Kluwer, Dordrecht), p. 27.

Fleming, W.P. 1912. Ann. Harv. Coll. Obs. 56, 165.

Garcia, M.R., Baliunas, S.L., Doxsey, R., Elvis, M., Fabbiano, G., Koenigsberger, G., Patterson, J., Schwartz, D., Swank, J., and Watson, M.G. 1983. A strophys. J., 267, 291.

Hack, M., and Selvelli, P.L. 1982. Astr. Astrophys., 107, 200.

Hogg, F.S. 1934. Publ. Am. Astr. Soc., 38, 14.

Iben, I. Jr., and Tutukov, A.V. 1984. A strophys. J. Suppl., 54, 335.

Isliker, H., Nussbaumer, H., and Vogel, M. 1989. Astr. Astrophys., $219,271$.

Kenyon, S.J. 1986. The Symbiotic Stars (Cambridge: Cambridge University Press).

Kenyon, S.J., Fernández-Castro, T., and Stencel, R.E. 1988. Astr. J., 95, 1817.

Kenyon, S.J., and Garcia, M.R. 1989. Astr. J., 97, 194.

Kenyon, S.J., Oliversen, N.A., Mikolajewska, J., Mikołajewski, M., Stencel, R.E., Garcia, M.R., and Anderson, C.M. 1990. Astr. J., 101, 637.

Kenyon, S.J., and Truran, J.W. 1983. A strophys. J., 273, 280.

Kenyon, S.J., and Webbink, R.F. 1984. Astrophys. J., 279, 252.

Kuiper, G.P. 1941. Astrophys. J., 93, 133.

Menzel, D.H. 1946. Physica, 12, 768.

Merrill, P.W., and Humason, M.L. 1932. Publ. Astr. Soc. Pac., 44, 56.

Mikołajewska, J., Friedjung, M., Kenyon, S.J., and Viotti, R. 1988. editors, The Symbiotic Phenomenon, IAU Colloquium No. 103, (Kluwer, Dordrecht).

Mikołajewska, J., and Kenyon, S.J. 1992a. Astr. J, submitted.

Mikołajewska, J., and Kenyon, S.J. 1992b. Mon. Not. Roy. Astr. Soc., submitted.

Mikołajewska, J., Kenyon, S.J., and Mikolajewski, M. 1989. Astr. J., 98, 1427.

Mürset, U., Nussbaumer, H., Schmid, H.M., and Vogel, M. 1991. Astr. Astrophys., , .

Nussbaumer, H., and Stencel, R.E. 1987. In Exploring the Universe with the IUE Satellite, edited by Y. Kondo (Reidel, Dordrecht), p. 203.

Nussbaumer, H., Schild, H., Schmid, H.M., and Vogel, M. 1988. Astr. Astrophys., $198,179$.

Nussbaumer, H., and Vogel, M. 1987. Astr. Astrophys., 182, 51.

Paczyński, B. 1976. Acta A str., 21, 417.

Payne-Gaposchkin, C. 1957. The Galactic Novae (Amsterdam: North Holland)

Savage, B.D., and Mathis, J.S. 1979. Ann. Rev. Astr. Ap., 17, 73.

Seaquist, E.R., and Taylor, A.R. 1990, Astrophys. J., 349, 313.

Tout, C.A., and Eggleton, P.P. 1988. Mon. Not. Roy. Astr. Soc., 231, 823.

van den Bergh, S., and Tammann, G.A. 1991. Ann. Rev. Astr. Ap., 29, 363.

Vogel, M. 1991. Astr. Astrophys., ,.

Wallerstein, G. 1981, Observatory, 101, 172.

Webster, B.L., and Allen, D.A. 1975. Mon. Not. Roy. Astr. Soc., 171, 171.

Whitelock, P.A. 1987. Publ. Astr. Soc. Pac., 99, 573.

Willson, L.A., Wallerstein, G., Brugel, E., and Stencel, R.E. 1984. Astr. Astrophys., 133, 154.

Wood, P.R. 1973, Publ. Astr. Soc. Aus., 2, 198. 